\title{
A Personalized Mean-CVaR Portfolio Optimization Model for Individual Investment
}

\author{
Chunxia Yu (D) $^{1}$ and Yuru Liu ${ }^{1,2}$ \\ ${ }^{1}$ School of Economics and Management, China University of Petroleum, Beijing, China \\ ${ }^{2}$ School of Economics and Management, Beijing University of Posts and Telecommunications, Beijing, China \\ Correspondence should be addressed to Chunxia Yu; yuchunxiasd@163.com
}

Received 10 September 2020; Revised 23 January 2021; Accepted 19 February 2021; Published 8 March 2021

Academic Editor: Zabidin Salleh

Copyright (c) 2021 Chunxia Yu and Yuru Liu. This is an open access article distributed under the Creative Commons Attribution License, which permits unrestricted use, distribution, and reproduction in any medium, provided the original work is properly cited.

\begin{abstract}
Investment as an important issue in daily life is accompanied by the occurrence of various financial assets, such as stocks, bonds, and mutual funds. However, risk tolerances vary across individuals. Individual investors have to select corresponding personalized investment portfolios to satisfy their own needs. Moreover, it is difficult for ordinary people to select a personalized investment portfolio by themselves, and it is too expensive and inefficient to look for professional consultation. Therefore, the objective of this research is to propose a personalized portfolio recommendation model, which can build the personalized portfolio based on investors' risk tolerances. In this research, investors' risk tolerance is determined by the fuzzy comprehensive evaluation method based on investors' demographic characteristics. The CVaR is used as the risk measurement of financial assets. The dynamics of the distribution of returns are described in the combined Copula-GARCH model, and the future scenarios of returns are generated by the Monte Carlo simulation based on the combined Copula-GARCH model to estimate CVaR. The mean-CVaR portfolio optimization model is used to find out the best personalized portfolio. Finally, experiments are conducted to validate the applicability and feasibility of the personalized investment portfolio optimization model. Results show that the proposed investment portfolio optimization model can recommend personalized investment portfolio according to investor's risk tolerance.
\end{abstract}

\section{Introduction}

In recent years, various types of financial assets are available for investors, such as stocks, bonds, and mutual funds. These investment vehicles, especially online financial assets, have provided new options for individuals. Accordingly, investment is not a theme which is only discussed by wealthy people and professional investors any more, it also involves common people. Risk tolerance is generally thought of as the level of financial risk that a client is willing to accept [1]. An individual's investment risk tolerance tends to influence his/ her investment preference or decision [2]. For this reason, investment portfolios should be personally configured for each individual. However, ordinary people have no specialized financial knowledge and cannot select personalized investment portfolios by themselves. Therefore, a personalized investment portfolio optimization model is necessary for individual investors to build the investment portfolio according to their risk tolerances.

Markowitz [3] first developed the idea of portfolio optimization, where expected return is maximized given some maximum allowed variance or variance is minimized subject to some minimum expected return. Markowitz [3] proposed the pioneering work for determining the optimal allocation rates of assets in a portfolio using the mean-variance model. In the mean-variance model, variance is the risk measure to estimate financial assets' market risk. Nevertheless, variance cannot define the direction of volatility [4]. Subsequently, Value-at-Risk (VaR) which is able to estimate the highest loss over a target horizon in the environment of the normal market is presented as another risk measure. Then, the mean VaR model is applied for portfolio optimization [4]. However, VaR is not subadditive and nor convex [5]. Furthermore, VaR is unable to give the details about 
measuring the losses in the tail [6]. Based on the concept of $\mathrm{VaR}$, Conditional Value-at-Risk ( $\mathrm{CVaR})$ was developed as a coherent risk measure. CVaR satisfies the axioms of translation invariance, subadditivity, positive homogeneity, and monotonicity [7]. Subsequently, more and more researchers adopted the mean-CVaR model in portfolio optimization applications [7]. Nevertheless, the existing optimization models only focused on financial assets' market risk and have not considered the personalized risk tolerances of individual investors.

Risk tolerance is the amount of uncertainty or investment return volatility that an investor can tolerate in financial investment [8], and it is an important issue that should be considered in investment. Therefore, the objective of this research is to propose a personalized portfolio recommendation model, which can build the personalized portfolio based on investors' risk tolerances. In this research, investors' risk tolerance is determined by the fuzzy comprehensive evaluation method based on investors' demographic characteristics. CVaR is used as the risk measurement of financial assets. The dynamics of the distribution of returns are described in the combined CopulaGARCH model, and the future scenarios of returns are generated by the Monte Carlo simulation based on the combined Copula-GARCH model to estimate CVaR. The mean-CVaR portfolio optimization model is used to find out the best personalized portfolio.

This research consists of five parts. A review of relevant risk tolerance, $\mathrm{CVaR}$, and mean-CVaR models is illustrated in Section 2. Section 3 proposes a portfolio optimization model for individual investors. Section 4 introduces the experiments conducted to validate the applicability and feasibility of the personalized mean-CVaR portfolio optimization model. Finally, Section 5 summarizes the research and expects to the further directions.

\section{Literature Review}

2.1. Investor Risk Tolerance. Risk tolerance is extensively used to represent investors' attitudes towards risk in the personal financial investment field [9-11]. Some studies obtained the relationship between risk tolerance and personal demographic characteristics [12-14]. They found that the relationship between demographic characteristic education and risk tolerance is positive. Similarly, the relationship between demographic characteristic investment knowledge with risk tolerance is positive. Nevertheless, demographic characteristic age has a negative relationship with risk tolerance. Besides, males have a higher degree of risk acceptability than females. Thus, demographic characteristics, gender, age, education, and investment knowledge can be used as criteria to assess investors' risk tolerance. Furthermore, the risk tolerance determination problem is regarded as a multicriteria decision-making problem that analyses and evaluates the performances of investors on demographic characteristics by decision-making approaches.

Among the approaches presented to calculate risk tolerance, the FCE is a multilayer comprehensive evaluation approach that deals with imprecise and uncertain data by fuzzy mathematics [15]. The FCE-based approach is widely used to estimate risk degrees comprehensively and scientifically in different applications [16-18]. Consequently, the FCE method is employed in this research to assess investors' risk tolerance.

2.2. An Overview of $C V a R$. CVaR is the average value of a loss over a certain threshold within a certain confidence interval affected by the tail of the distribution [19]. It satisfies the properties of a coherent risk measure [20]. When calculating $\mathrm{CVaR}$, researchers usually assume that asset returns have normal distributions and linear relationships [21]. However, in practice, asset returns do not follow normal distribution, and the relationships between asset returns are time-varying and nonlinear [22]. Therefore, modeling the dependence structure between financial asset returns is necessary to estimate portfolio CVaR.

The GARCH model presented by Bollerslev [23] is an efficient way to estimate financial instrument return and volatility under a more real-world condition [24, 25]. Hence, in this research it has the capacity to estimate marginal distributions of the financial asset. In addition, the Copula model has the capacity to determine the correlation between variables, in which the multivariate joint distribution function is written by univariate marginal distributions [26]. Hence, the Copula model is able to model the dependence structure between financial asset returns with nonnormal distribution and nonlinear relationship [21, 27]. Thus, this research uses the combined Copula-GARCH model to construct the dynamics of the distribution of returns, with the GARCH model estimating asset returns and the Copula model constructing the dependence structure across asset returns.

Three ways are commonly used to measure CVaR, namely, the parametric approach, the historical method, and the Monte Carlo simulation [4]. To start with, the parametric approach assumes that the distribution function of financial asset return is known. The historical simulation approach uses historical data directly. The Monte Carlo simulation approach simulates several random scenarios [28, 29]. In this study, the Monte Carlo simulation is adopted to generate the future return rate sequence of financial assets based on the combined Copula-GARCH model.

\subsection{An Overview of Mean-CVaR Models. Different mean-} $\mathrm{CVaR}$ models were proposed for portfolio optimization problems [5, 30, 31]. Researchers viewed mean-CVaR models as a multiobjective optimization problem minimizing the value of $\mathrm{CVaR}$ under a known expected return or maximizing the expected return under a known expected CVaR. Moreover, different intelligent algorithms have been used to solve mean-CVaR models [31-33]. The heuristic algorithm PSO is simple and accessible [34] and does not involve many parameters to be adjusted [35]. Consequently, the PSO algorithm is adopted in this study to determine the optimal investment portfolio for the personalized meanCVaR portfolio optimization model. 


\section{A Personalized Mean-CVaR Portfolio Optimization Model}

A mean-CVaR model incorporating investors' risk tolerance is formulated to provide a personalized portfolio for individual investors. The first step is to categorize investor's risk tolerance. The next step is to compute portfolio CVaR by the combined Copula-GARCH model and Monte Carlo simulation. The final step is to formulate the mean-CVaR portfolio optimization model incorporating investors' risk tolerances and determine the optimal investment portfolio by the PSO algorithm. Figure 1 shows the framework of the personalized mean-CVaR portfolio optimization model, and concrete information will be presented in the following subsections.

3.1. Investor Risk Tolerance Determination. This section is going to evaluate risk tolerance of investors and to divide it into the corresponding risk levels based on the experts' weights and FCE-integrated method.

3.1.1. Risk Tolerance Determination Method. The FCE method is a mathematical approach to evaluate things comprehensively based on fuzzy mathematics methods [36]. The experts' weights and FCE-integrated method for investor risk tolerance determination are summarized as follows:

Step 1: formulate risk tolerance evaluation criteria

As was mentioned in Section 2.1, the demographic characteristics such as gender, age, education, investment experience, and income shown in Table 1 are adopted as evaluation criteria of risk tolerance. The relationship between education levels and risk tolerance is positive, relationship between investment knowledge and risk tolerance is positive, while relationship between age and risk tolerance is negative. Moreover, males have a higher degree of risk acceptability than females. Then, the evaluation criteria set \{gender, age, education, investment knowledge $\}$ is formulated and is denoted by $H=\left\{h_{1}, h_{2}, \ldots, h_{l}\right\}$, where $l$ equals 4 .

Step 2: determine weights of risk tolerance evaluation criteria

The weights of risk tolerance evaluation criteria $W^{r}=$ $\left(w_{1}^{r}, w_{2}^{r}, \ldots, w_{l}^{r}\right)$ are determined based on experts' opinions, and $\sum_{i=1}^{l} w_{i}^{r}=1$.

Step 3: determine investor's risk level

The risk tolerance of an investor ranges from the conservative to the aggressive level. It can be divided it into five levels, namely, conservative (C) level, moderately conservative (MC) level, moderate (M) level, moderately aggressive (MA) level, and aggressive (A) level. Then, the risk level set $G=\left\{g_{1}, g_{2}, \ldots, g_{f}\right\}$ is established where $f$ equals 5 .

Step 4: determine fuzzy relationship matrix

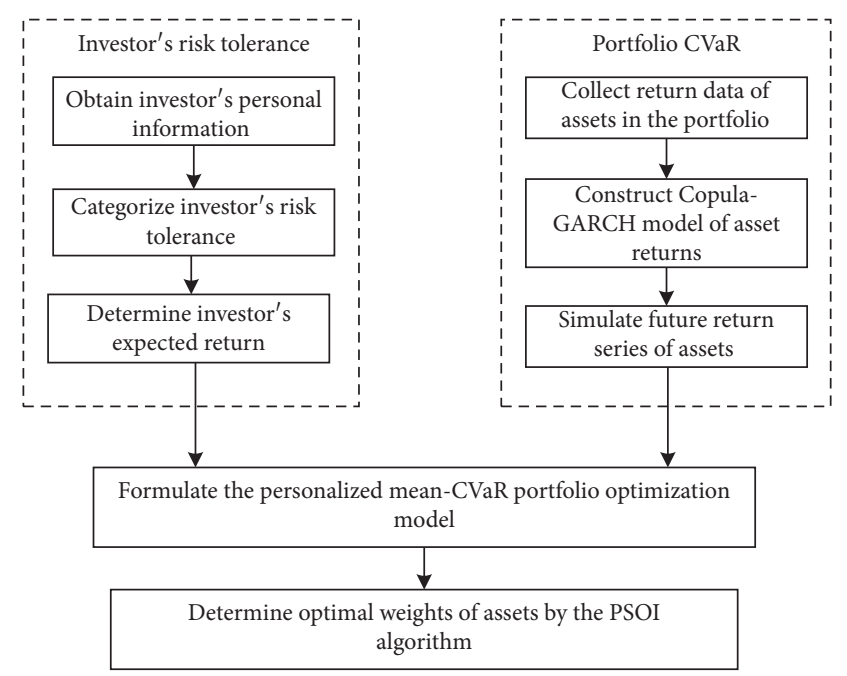

FIGURE 1: Framework of the personalized mean-CVaR portfolio optimization model.

The fuzzy relationship matrix $R$ shown as equation (1) is determined by the decision-maker, where $q_{i j}$ represents the $i^{\text {th }}$ criterion's membership degree belonging to the $j$ th risk level:

$$
R=\left(q_{i j}\right)_{l \times f}=\left[\begin{array}{cccc}
q_{11} & q_{12} & \cdots & q_{1 f} \\
q_{21} & q_{22} & & q_{2 f} \\
\vdots & \vdots & \ddots & \vdots \\
q_{l 1} & q_{l 2} & \cdots & q_{l f}
\end{array}\right] .
$$

Step 5: determine the comprehensive evaluation category

The comprehensive evaluation vector $B=\left(b_{1}, b_{2}, \ldots, b_{f}\right)$ is determined as equation (2), by compositing the weights of evaluation criteria and the fuzzy relationship matrix:

$$
\begin{aligned}
B & =W^{r} \times R_{i j}=\left(w_{1}^{r}, w_{2}^{r}, \ldots, w_{l}^{r}\right)\left[\begin{array}{cccc}
q_{11} & q_{12} & \cdots & q_{1 f} \\
q_{21} & q_{22} & & q_{2 f} \\
\vdots & \vdots & \ddots & \vdots \\
q_{l 1} & q_{l 2} & \cdots & q_{l f}
\end{array}\right] \\
& =\left(b_{1}, b_{2}, \ldots, b_{f}\right) .
\end{aligned}
$$

Then, the final risk tolerance degree $d \in[0,1]$ is determined bythe following equation:

$$
d=E \times B=e_{1} \times b_{1}+\cdots+e_{j} \times b_{j}+\cdots+e_{f} \times b_{f},
$$

where $E=\left\{e_{1}, e_{2}, \ldots, e_{f}\right\}$ denotes risk tolerance degrees of each risk level which can be assigned by the decision-maker.

In this research, the authors assume five risk tolerance levels, namely, $\{\mathrm{C}, \mathrm{MC}, \mathrm{M}, \mathrm{MA}, \mathrm{A}\}$, so five weights according to the risk tolerance level from low to high are designed as $\{0.1,0.2,0.3,0.4,0.5\}$. These weights are used to determine 
TABLE 1: Risk tolerance evaluation criteria.

\begin{tabular}{lcc}
\hline Criterion & Illustration & Value range \\
\hline Gender & $\begin{array}{c}\text { Males have a higher } \\
\text { degree of risk } \\
\text { acceptability than } \\
\text { females }\end{array}$ & Male, female \\
\hline Age & Negative relationship & $\begin{array}{c}<30 \text { years old, 31-40 } \\
\text { old, } 51-60 \text { years old, } \\
>60 \text { years old }\end{array}$ \\
\hline Education & Positive relationship & $\begin{array}{c}\text { Did not complete high } \\
\text { school, high school, } \\
\text { university }\end{array}$ \\
\hline $\begin{array}{l}\text { Investment } \\
\text { knowledge }\end{array}$ & Positive relationship & $\begin{array}{c}\text { Not rich, moderate, } \\
\text { rich }\end{array}$ \\
\hline
\end{tabular}

the overall risk tolerance evaluation index. When it is in the situation of $0.4<d \leq 0.52$, the investor is conservative; when $0.52<d \leq 0.64$ is satisfied, the investor belongs to the moderately conservative level; when $0.64<d \leq 0.76$ is guaranteed, the investor is moderate; when $0.76<d \leq 0.88$ is provided, the investor is moderately aggressive; given $0.88<d \leq 1$, the investor is aggressive.

3.1.2. Risk Tolerance Level Analysis. The risk level investors can tolerate and the rates of return investors want are different. For the five risk levels introduced in Section 3.1.1, their risk levels and expected returns are explained as follows.

Aggressive and moderately aggressive investors have higher risk tolerance, and they can accept the possible volatility. The objective of them is the high growth of funds. They expect a higher rate of return than the general interest rate in the market.

Moderate investors also can bear a certain level of loss, but they have relatively lower risk tolerance than aggressive and moderately aggressive investors. The objective of moderate investors is asset preservation rather than high return. They expect a general interest rate in the current market.

Conservative and moderately conservative investors have extremely low risk tolerance and requirements on the benefit. They are in pursuit of absolute security of capital and ensure the principal in the inflation protection. Their expected return rate is slightly better than the short- and medium-term deposit rate.

In view of this, the specific matching rules between the risk level of investors and expected return are shown in Table 2, under that the general interest rate in the current market is $7 \%$ and one-year deposit rate is about $4 \%$ from the report of People's Bank of China.

3.2. Computation of CVaR. CVaR is employed in this research as the risk measurement of financial assets. In this study, the dynamics of the distribution of returns are described in the combined Copula-GARCH model, and the future scenarios of returns are generated by the Monte Carlo simulation based on the combined Copula-GARCH model to estimate CVaR.
TABLE 2: Matching rules between investor's risk tolerance level and expected return.

\begin{tabular}{lcc}
\hline Risk level & $\begin{array}{c}\text { Annual expected } \\
\text { return } r(\%)\end{array}$ & $\begin{array}{c}\text { Daily expected return } \\
r_{0}=r / 365(\%)\end{array}$ \\
\hline A & 14 & 0.0384 \\
MA & 10 & 0.0274 \\
M & 7 & 0.0192 \\
MC & 4 & 0.0120 \\
C & 3 & 0.0822 \\
\hline
\end{tabular}

3.2.1. Definition of $C V a R$. VaR is a risk measure that describes the highest loss of an asset within a certain confidence interval. The definition can be described as follows:

$$
\int_{-\infty}^{-\mathrm{VaR}} p(x)=1-\alpha
$$

where $\alpha(0<\alpha<1)$ is the confidence interval decided by the decision-maker, $x$ denotes the variation for the asset return, and $p(x)$ denotes the probability density function of the variation for the asset return. Then, $\mathrm{VaR}_{\alpha}$ represents the highest loss at the confidence interval.

The CVaR is an improved coherent risk measure, and it considers the expected loss below the VaR [16]. Then, CVaR can be expressed as follows:

$$
\mathrm{CVaR}=E\left(x \mid x \geq \operatorname{VaR}_{\alpha}\right) .
$$

Then, the CVaR also can be represented as follows:

$$
\mathrm{CVaR}_{\alpha}=(1-\alpha)^{-1} \int_{-\infty}^{-\mathrm{VaR}_{\alpha}}-x p(x) \mathrm{d} x .
$$

In the research, the return distribution is determined by the historical return performance, and the future return series is simulated based on the determined return distribution. Let discrete set $Q=\left(s_{1}, s_{2}, \ldots, s_{n}\right)$ denotes future scenarios, $\pi_{j}$ represents the probability of scenario $s_{j}$ occurring, and $\sum \pi_{j}=1$.Then, the CVaR also can be described as follows [6]:

$$
\mathrm{CVaR}_{\alpha}(c)=c+(1-\alpha)^{-1} \sum_{j=1}^{n} \pi_{j}\left(-x_{j}-c\right)^{+},
$$

where $(D)^{+}=\max \{D, 0\}, c$ is another form of $\operatorname{VaR}_{\alpha}$, $P\left(-x_{j} \geq c\right)=1-\alpha$, and $x_{j}$ denotes the variation for the portfolio return in scenario $s_{j}$.

3.2.2. Estimation of Distribution of Returns Using the CopulaGARCH Model. In this research, the GARCH model describes the time series of returns, and the Copula model estimates the dependence structures between return series. Engle [37] proposed the LM test method for the ARCH process, which can be used to judge whether there is an $\mathrm{ARCH}$ effect in the financial sequence. If there is an $\mathrm{ARCH}$ effect, the GARCH model can be used for estimation. At present, the GARCH $(1,1)$ model is widely used to describe the volatility and CVaR of financial assets [38]. The conditional distribution of the financial return series has the characteristics of fluctuation, skewness, leptokurtosis, and thick tail. Therefore, the $T$ distribution with leptokurtosis and thick tails is proposed to replace the normal distribution. The 
time series of return are characterized by heteroscedasticity, leptokurtosis, and leverage effect, so the $t$-GARCH $(1,1)$ is adopted to estimate marginal distribution of return series.

Then, the return of the financial asset can be defined as follows:

$$
\begin{aligned}
r_{t i} & =u_{i}+a_{t i}, \\
a_{t i} & =\sigma_{t i} \times \varepsilon_{t i}, \\
\sigma_{t i}^{2} & =\beta_{t 0}+\beta_{t 1} \times a_{t-1, i}^{2}+\beta_{t 2} \times \sigma_{t-1, i}^{2}, \\
\varepsilon_{t i} & \sim t\left(\theta_{i}\right),
\end{aligned}
$$

where $r_{t i}, t=1,2, \ldots, T$ represents actual return of the $i^{\text {th }}$ asset, $u_{i}$ denotes the estimated return of the $i^{\text {th }}$ asset which is estimated based on historical data, and $a_{t i}$ represents the difference between $r_{t i}$ and $u_{i}$. In the GARCH model, $a_{t i}$ can be divided into the volatility of return $\sigma_{t i}$ and the random error $\varepsilon_{t i}$, and $\beta_{0 i}, \beta_{1 i}$, $\beta_{2 i}$, and $\theta_{i}$ are parameters. In this research, we assume that $\varepsilon_{t i}$ follows Student's $t$-distribution, and the parameters in the GARCH model can be calculated by Eviews software.

Under the assumption that the dependence structures between return series do not vary with time, the $t$-Copula model is adopted to describe the tail risk of assets. Then, the $t$-Copula function could be written as follows [39]:

$$
C_{\lambda, \Sigma}^{t}\left(y_{t 1}, y_{t 2}, \ldots, y_{t m}\right)=t_{\lambda, \Sigma}\left(t_{\theta 1}^{-1}\left(y_{t 1}\right), t_{\theta 2}^{-1}\left(y_{t 2}\right), \ldots, t_{\theta m}^{-1}\left(y_{t m}\right)\right),
$$

where $y_{t i}$ means the variable in the model, $t_{\lambda, \Sigma}$ denotes the multivariate $t$-distribution with the correlation matrix $\Sigma$ and degree of freedom $\lambda$, and $t_{\theta}^{-1}$ represents the inverse of the cumulative $t$-distribution.

Assume that a sample $\varepsilon_{t}=\left\{\varepsilon_{t 1}, \varepsilon_{t 2}, \ldots, \varepsilon_{t m}\right\}$ follows Student's $t$-distribution and the degree of freedom is $\theta=\left\{\theta_{1}, \theta_{2}, \ldots, \theta_{m}\right\}$, where $t=1, \ldots, T$, then

$$
y_{t}=\left(y_{t 1}, y_{t 2}, \ldots, y_{t m}\right)=\left(t_{\theta 1}\left(\varepsilon_{t 1}\right), t_{\theta 2}\left(\varepsilon_{t 2}\right), \ldots, t_{\theta m}\left(\varepsilon_{t m}\right)\right) \text {. }
$$

Then, the joint distribution function $F\left(\varepsilon_{t}\right)$ can be obtained by the following equation:

$$
F\left(\varepsilon_{t 1}, \varepsilon_{t 2}, \ldots, \varepsilon_{t m}\right)=C_{\lambda, \Sigma}^{t}\left(t_{\theta 1}\left(\varepsilon_{t 1}\right), t_{\theta 2}\left(\varepsilon_{t 2}\right), \ldots, t_{\theta m}\left(\varepsilon_{t m}\right)\right) .
$$

Combining equations (8)-(11), the Copula-GARCH model is formulated as follows:

$$
\begin{aligned}
r_{t i} & =u_{i}+a_{t i}, \\
a_{t i} & =\sigma_{t i} \times \varepsilon_{t i}, \\
\sigma_{t i}^{2} & =\beta_{t 0}+\beta_{t 1} \times a_{t-1, i}^{2}+\beta_{t 2} \times \sigma_{t-1, i}^{2}, \\
\varepsilon_{t i} & \sim t\left(\theta_{i}\right), \\
F\left(\varepsilon_{t 1}, \varepsilon_{t 2}, \ldots, \varepsilon_{t m}\right) & =C_{\lambda, \Sigma}^{t}\left(t_{\theta 1}\left(\varepsilon_{t 1}\right), t_{\theta 2}\left(\varepsilon_{t 2}\right), \ldots, t_{\theta m}\left(\varepsilon_{t m}\right)\right) .
\end{aligned}
$$

3.2.3. Simulation of Future Returns. The future scenarios of returns can be generated by the Monte Carlo simulations based on the results of the combined Copula-GARCH model by using the following procedure:

(1) Determine the parameters in equation (8) based on the historical return series from time 0 to time $T$ using Eviews

(2) Obtain the joint distribution function $C_{\lambda, \Sigma}^{t}$ with equation (9) based on the historical data of random error from time 0 to time $T$ and the parameter $\theta_{i}$

(3) Generate $q$ random variables $\left(u_{1}, u_{2}, \ldots, u_{q}\right)$ based on the obtained $C_{\lambda, \Sigma}^{t}$

(4) Get random disturbances $\left(\xi_{1}, \xi_{2}, \ldots, \xi_{q}\right)$ based on the $t$-distribution function estimated in the GARCH model as

$$
\left(\xi_{1}, \xi_{2}, \ldots, \xi_{q}\right)=\left(t_{\theta 1}^{-1}\left(u_{1}\right), t_{\theta 2}^{-1}\left(u_{2}\right), \ldots, t_{\theta q}^{-1}\left(u_{q}\right)\right)
$$

(5) Calculate the future return rate sequence of the assets at time $T+1$ based on estimated equation (8)

(6) Obtain the given number of possible scenarios by repeating steps (3)-(5)

3.3. The Proposed Mean-CVaR Model. The personalized mean-CVaR portfolio optimization model is to select optimal weights of each asset that minimize the risk level measured by CVaR under a known expected return. The mean-CVaR model considering investor's risk tolerance is proposed, and the optimal investment portfolio is determined by the PSO algorithm.

3.3.1. Formulation of the Personalized Mean-CVaR Model. We assume that $m$ types of financial assets are available in the market, $n$ kinds of scenarios $Q=\left(s_{1}, s_{2}, \ldots, s_{n}\right)$, and the occurrence probability of each scenario $s_{j}$ is the same. Since the expected return can be determined for each risk level investor by the method proposed in Section 3.1, the objective of the proposed personalized mean-CVaR model is to minimize the $\mathrm{CVaR}_{\alpha}$. Subsequently, the portfolio optimization model incorporating investors' risk tolerance is formulated as the following:

$$
\begin{array}{ll}
\min \quad & \mathrm{CVaR}_{\alpha}=c+(1-\alpha)^{-1} n^{-1} \sum_{j=1}^{n} \tau_{j}, \\
& \sum_{i=1}^{m} w_{i} E\left(r_{i}\right) \geq r_{o}, \\
& \sum_{i=1}^{m} w_{i}=1, \\
& w_{i} \geq 0, \quad i=1,2, \ldots, m, \\
\text { s.t. } & E\left(r_{i}\right)=n^{-1} \sum_{j=1}^{n} r_{i j}, \\
& \tau_{j} \geq\left(f\left(w, r_{j}\right)-c\right)^{+}, \\
& \tau_{j} \geq 0, \quad j=1,2, \ldots, n, \\
& f\left(w, r_{j}\right)=\sum_{i=1}^{m}-w_{i} r_{i j},
\end{array}
$$


where $w_{i}$ represents the relative weight of the $i^{\text {th }}$ financial asset, column vector $r_{j}=\left(r_{1 j}, \ldots, r_{i j}, \ldots, r_{n j}\right)$ denotes asset returns at the scenario $s_{j}$, row vector $r_{i}=\left(r_{i 1}, \ldots\right.$, $\left.r_{i j}, \ldots r_{i m}\right)^{T}$ represents possible returns of the $i^{\text {th }}$ asset, $r_{o}$ refers to the given expected return, and $f\left(w, r_{j}\right)$ means the loss beyond $c$ of the portfolio at scenario $s_{j}$.

By the risk tolerance determination method proposed in Section 3.1, the risk tolerance of investors can be placed in five levels. Once the investor's risk preference is known, the parameters $\alpha$ and $r_{o}$ in the proposed mean-CVaR model can be determined by Table 3 . Then, the time series of return is estimated by the GARCH model, and the joint distributions of returns between assets are described by the Copula model based on historical data. Then, the future scenarios of returns are generated by the Monte Carlo simulation based on the results of the combined Copula-GARCH model. Consequently, the proposed mean-CVaR model is able to obtain the optimal weights of assets and recommend suitable portfolios to investors according to the future scenarios of returns.

3.3.2. PSO-Based Optimal Portfolio Determination Algorithm. PSO algorithm is adopted in this study to compute the optimal portfolio for individual investors, namely, determine optimal weights of financial assets in a portfolio. Firstly, particles representing possible solutions are initialized randomly, and then, the optimal solution is searched by particles' iterations.

In this paper, the position vector is $\left(\omega_{p 1}, \omega_{p 2}, \ldots\right.$, $\left.\omega_{p i}, \ldots, \omega_{p m}\right)$, where $\omega_{p i}$ denotes the relative weight of the $i^{\text {th }}$ financial asset for $p$ th iterations, $m$ is the number of financial assets, and $\sum_{i=1}^{m} \omega_{p i}=1$. The velocity vector is $\left(v_{p 1}, v_{p 2}, \ldots, v_{p i}, \ldots, v_{p m}\right)$, where $v_{p i}$ represents the velocity of $\omega_{p i}$ and $\sum_{p=1}^{n} v_{i p}=0$. The fitness function is defined as follows:

$$
\text { fitness value }=\left\{\begin{array}{l}
\mathrm{CVaR}, \quad \text { otherwise } \\
10, \quad W^{T} \bar{r}>r_{o} \text { or } w_{p i}<0 .
\end{array}\right.
$$

In equation (15), CVaR is calculated by equation (14), and $W=x_{i}$ denotes the weights of financial assets in the portfolio. Consequently, the updating rule is the smaller the fitness value the better. To sum up, the concrete procedure of the PSO algorithm to determine the relative weights of each asset in a portfolio is shown in Figure 2.

\section{Experiments and Results Analysis}

In the simulation, six financial assets are selected from three common types of assets, including one national debt (China debt 0303), three stocks (Apple Inc., Suning Inc., and China National Petroleum Corporation), and two funds (EEM (iShares MSCI Emerging Markets ETF) and SPY (SPDR S\&P 500 ETF)). The dataset is obtained from Wind and collected from 01/05/2015-28/04/2018 without data missed over holidays. For example, the authors assume that an individual investor who is a 35-year-old undergraduate woman with little investment knowledge looks for a personalized investment portfolio. Then, the proposed model is conducted to recommend a suitable optimal investment portfolio for this investor.

\subsection{Experiment 1: Application of the Personalized Portfolio Optimization Model.}

Phase 1: determination of investors' risk tolerance

Step 1: the weights of evaluation criteria, as shown in Table 4, are gained based on expert opinions

Step 2: given investor's fuzzy relationship matrix can be determined by Table 3 and equation (1) as follows:

$$
R=\left[\begin{array}{ccccc}
1 & 0.75 & 0.5 & 0.25 & 0 \\
0.25 & 0.5 & 0.75 & 1 & 0.75 \\
0 & 0.25 & 0.5 & 0.75 & 1 \\
1 & 0.75 & 0.5 & 0.25 & 0
\end{array}\right]
$$

Step 3: the comprehensive evaluation vector is obtained by equation (2) as follows:

$$
\begin{aligned}
B & =(0.2,0.35,0.25,0.2) R \\
& =\left[\begin{array}{ccccc}
1 & 0.75 & 0.5 & 0.25 & 0 \\
0.25 & 0.5 & 0.75 & 1 & 0.75 \\
0 & 0.25 & 0.5 & 0.75 & 1 \\
1 & 0.75 & 0.5 & 0.25 & 0
\end{array}\right] \\
& =(0.4875,0.5375,0.5875,0.6375,0.5125) .
\end{aligned}
$$

Step 4: the judgment value is obtained by equation (3) as follows:

$$
\begin{aligned}
d= & 0.1 \times 0.4875+0.2 \times 0.5375+0.3 \\
& \times 0.5875+0.4 \times 0.6375+0.5 \times 0.5125, \\
= & 0.84375
\end{aligned}
$$

Since $0.64<d \leq 0.88$ is provided, the investor is moderately aggressive. According to the investor's risk preference and the matching rules between the risk level of investor and expected return shown in Table 3, the expected return for the investor is defined by $r_{0}=0.0274 \%$.

Phase 2: computation of CVaR

Log returns are widely used for stock returns. On the one hand, the log return can make the data more stable. On the other hand, the log return is additive so that the log returns of a single period can be added to get the overall log return. In this phase, the log return series for the six financial assets are determined by equation (19), where $p_{t}$ denotes daily closing prices:

$$
r_{t}=\ln \left(p_{t}\right)-\ln \left(p_{t-1}\right)
$$

Step 1: six GARCH models are created to describe the log return series of the six financial assets.

(1) The statistical characteristics of the historical log return series of the six financial assets are analyzed 
TABLE 3: Fuzzy relationship function knowledge base.

\begin{tabular}{|c|c|c|c|c|c|c|}
\hline Index & Value & $\mathrm{C}$ & $\mathrm{MC}$ & $\mathrm{M}$ & MA & A \\
\hline \multirow{2}{*}{ Gender } & Male & 0 & 0.25 & 0.5 & 0.75 & 1 \\
\hline & Female & 1 & 0.75 & 0.5 & 0.25 & 0 \\
\hline \multirow{5}{*}{ Age } & $<30$ & 0 & 0.25 & 0.5 & 0.75 & 1 \\
\hline & $31-40$ & 0.25 & 0.5 & 0.75 & 1 & 0.75 \\
\hline & $41-50$ & 0.5 & 0.75 & 1 & 0.75 & 0.5 \\
\hline & $51-60$ & 0.75 & 1 & 0.75 & 0.5 & 0.25 \\
\hline & $>60$ & 1 & 0.75 & 0.5 & 0.25 & 0 \\
\hline \multirow{3}{*}{ Education } & $<$ High school & 1 & 0.75 & 0.5 & 0.25 & 0 \\
\hline & High school & 0.5 & 0.75 & 1 & 0.75 & 0.5 \\
\hline & University & 0 & 0.25 & 0.5 & 0.75 & 1 \\
\hline \multirow{3}{*}{ Investment knowledge } & Not rich & 1 & 0.75 & 0.5 & 0.25 & 0 \\
\hline & Moderate & 0.5 & 0.75 & 1 & 0.75 & 0.5 \\
\hline & Rich & 0 & 0.25 & 0.5 & 0.75 & 1 \\
\hline
\end{tabular}

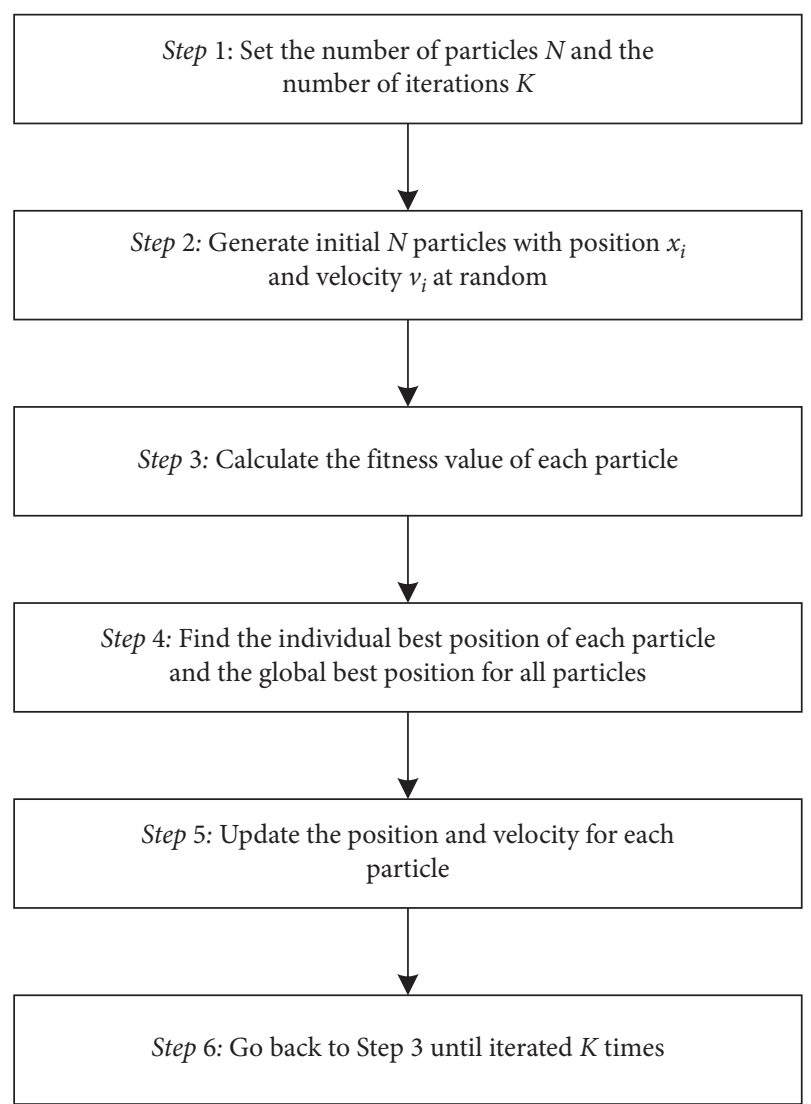

FIGURE 2: The procedure of the PSO algorithm for the proposed mean-CVaR model.

TABLE 4: Fuzzy relationship function.

\begin{tabular}{lcccc}
\hline Index & Gender & Age & Education & Investment knowledge \\
\hline Weights & 0.2 & 0.35 & 0.25 & 0.2 \\
\hline
\end{tabular}

by Eviews. Figures $3-8$ and Table 5 present the statistical characteristics and corresponding graphs of the six financial assets. The series of log returns of the six financial assets is shown to fluctuate up and

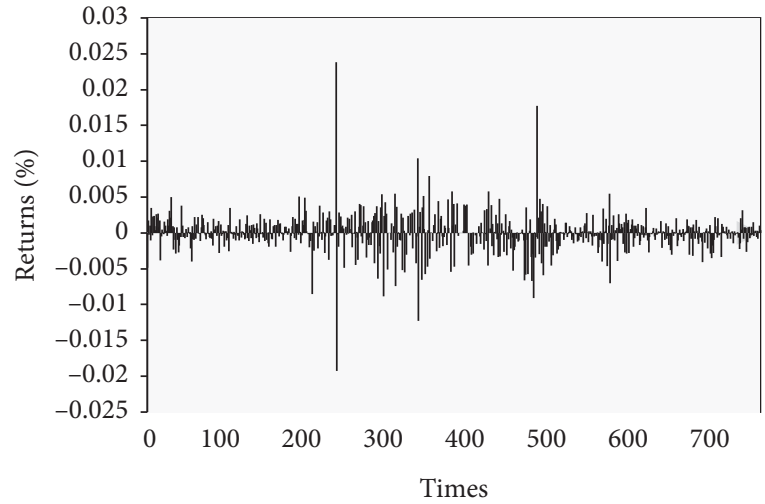

Figure 3: The China debt 0303 's daily log returns series.

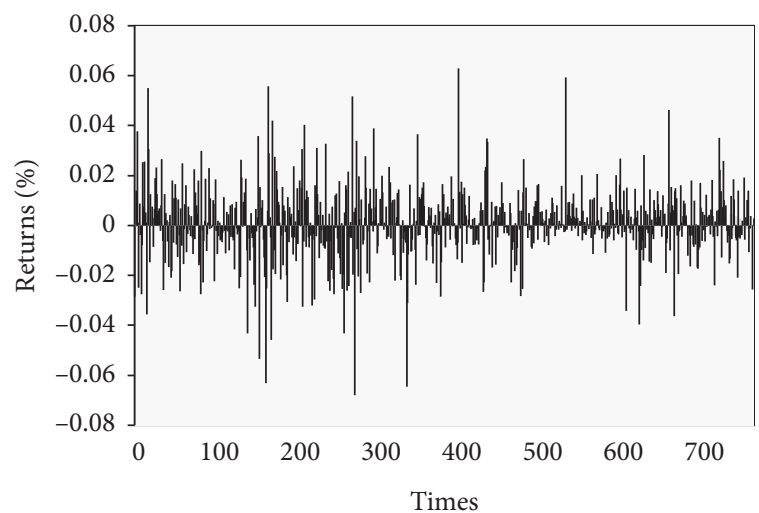

Figure 4: The Apple Inc.'s daily log return series.

down around 0 , and the degree of fluctuation is revealed to be different from different time periods. The $p$ value means that the probability that the J-B statistic exceeds the observed value under the null hypothesis. As shown in Table 5, the $p$ value of all series is almost zero, so the null hypothesis is rejected, and the series is not subject to normal distribution.

(2) The ARCH-LM test on the log return rate series is performed to test whether the return-on-asset ratio 


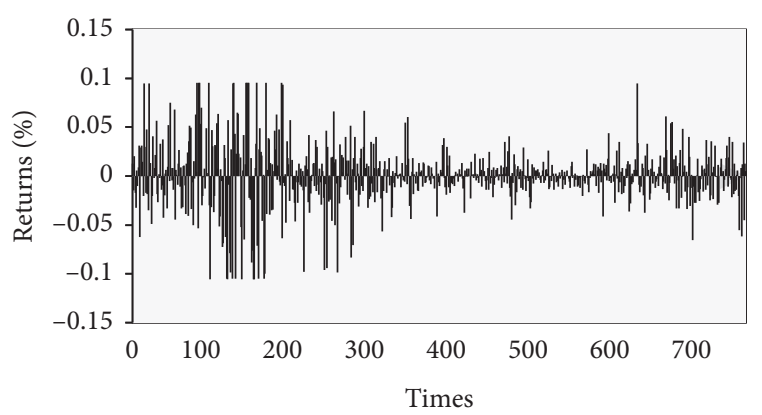

FIGURE 5: The Suning Inc.'s daily log return series.

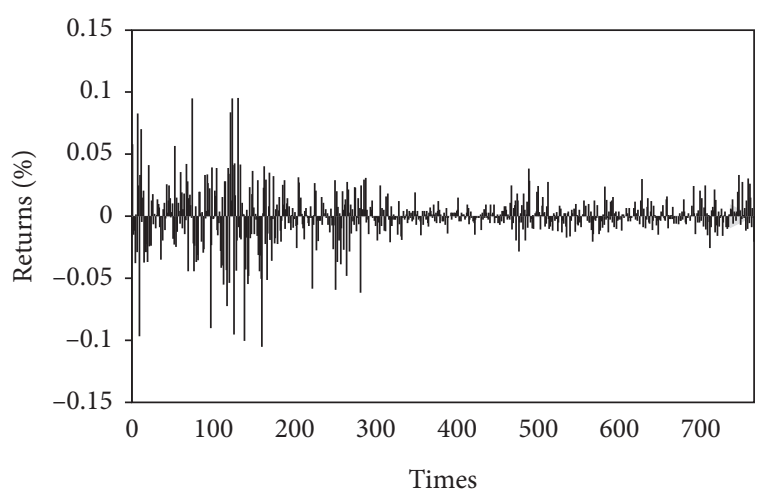

Figure 6: The CNPC's daily log return series.

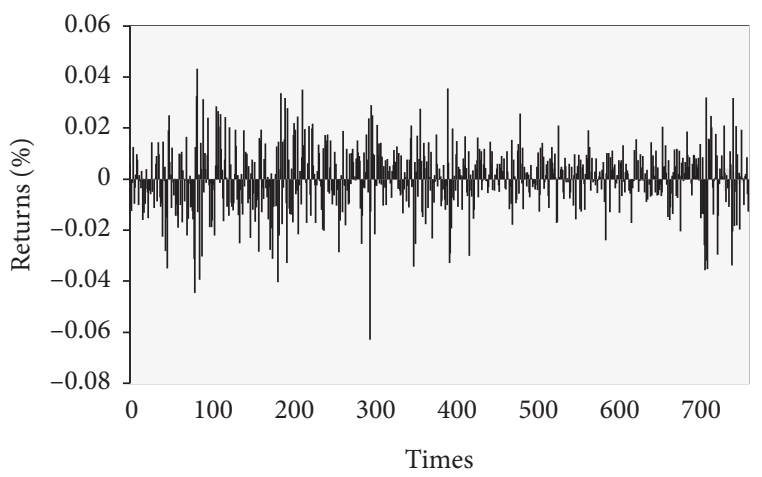

FIgURE 7: The EEM's daily log return series.

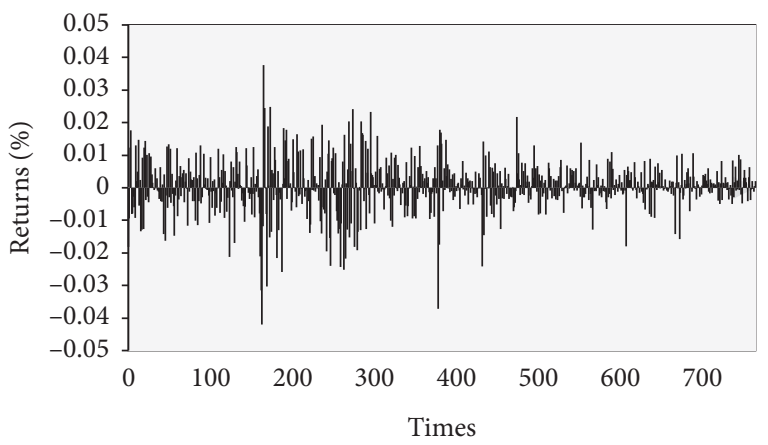

FIgURE 8: The SPY's daily log return series. has the ARCH effect. As shown in Table 6, the value of Prob is less than 0.01 , and thus, the assumption that $\mathrm{ARCH}$ exists and cannot be rejected. Therefore, the return series of the above six financial assets can be described by the GARCH model.

(3) Parameters for marginal distributions of the six financial assets, as shown in Table 7 , can be estimated by the $t$-GARCH $(1,1)$ model.

Step 2: a $t$-Copula model is established to describe the dependence structures between log return series. The parameters of the $t$-Copula function include the correlation coefficient matrix $\Sigma$ and the degree of freedom $\lambda$. The random disturbance term $\{\xi\}$ is calculated from the difference between the actual value and expected value, and then, the correlation coefficient matrix $\Sigma$ and the degree of freedom $\lambda$ of $\{\xi\}$ are calculated by the MATLAB. The correlation coefficient matrix is obtained, as shown in Table 8 , and the degree of freedom is 73.0058 . Then, all the parameters involved in the CopulaGARCH model are estimated.

Step 3: the future yields based on the CopulaGARCH model are simulated by Monte Carlo simulation.

(1) The parameters in the GARCH model have been determined by Step 1

(2) The parameters in the Copula model have been determined by Step 2

(3) Six random variables $\left(u_{1}, u_{2}, u_{3}, u_{4}, u_{5}, u_{6}\right)$ are generated

(4) The random disturbances based their $t$-distribution function are obtained. $\left(\xi_{1}, \xi_{2}, \xi_{3}, \xi_{4}, \xi_{5}, \xi_{6}\right)=$ $\left(t_{\theta 1}^{-1}\left(u_{1}\right), t_{\theta 2}^{-1}\left(u_{2}\right), t_{\theta 3}^{-1}\left(u_{3}\right), t_{\theta 4}^{-1}\left(u_{4}\right), t_{\theta 5}^{-1}\left(u_{5}\right), t_{\theta 6}^{-1}\left(u_{6}\right)\right)$

(5) The future log return-rate sequence of the assets at time $T+1$ is calculated based on estimated equation (8)

(6) One thousand scenarios, as shown in Table 9, are generated

Phase 3: determination of optimal portfolio

Step 1: the portfolio optimization model of the six assets is constructed based on the simulated future rate of $\log$ return under the condition that $\alpha=95 \%$. Step 2: the PSO algorithm with $N=200$ and $K=100$ is used to solve the portfolio optimization model. As shown in Figure 9, with the number of iterations increasing, CVaR gradually decreases and ultimately becomes stable. The optimal investment strategy is obtained by the PSO algorithm, and weights of each financial asset are $\{0.2199,0.1008,0.0020,0.0208$, $0.0429,0.6139\}$ with $\mathrm{CVaR}$ at 0.0081 .

As shown in Table 5, the mean value of expected log return of the six financial assets are $\{0.000002,0.000595$, $0.000334,-0.000354,0.000121,0.000353\}$. Then, the expected log return of the recommended investment portfolio is obtained by the weights of each financial asset and corresponding expected log return as following: 
TABLE 5: The descriptive statistics of the log returns of the six financial assets.

\begin{tabular}{lcccccccc}
\hline Sample & Mean & Maximum & Minimum & Std. dev. & Skewness & Kurtosis & J-B & $p$ value \\
\hline China Bonds 0303 & 0.000002 & 0.023828 & -0.019260 & 0.002477 & 0.686283 & 23.57103 & 13318.27 & 0 \\
Apple & 0.000595 & 0.062940 & -0.067965 & 0.014439 & -0.129436 & 6.232126 & 329.4278 & 0 \\
Suning & 0.000334 & 0.095569 & -0.105637 & 0.031149 & -0.171795 & 5.943309 & 275.1418 \\
CNPC & -0.000354 & 0.095384 & -0.105244 & 0.019322 & -0.322885 & 10.43322 & 1744.322 & 0 \\
EEM & 0.000121 & 0.043297 & -0.062929 & 0.012388 & -0.366746 & 4.535531 & 90.73705 & 0 \\
SPY & 0.000353 & 0.037675 & -0.042008 & 0.007835 & --0.464336 & 6.575980 & 427.7021 & 0 \\
\hline
\end{tabular}

TABLE 6: ARCH test results for the six financial assets.

\begin{tabular}{lll}
\hline ARCH-LM (1) & Q-stat & Prob \\
\hline China Bonds 0303 & 90.646 & 0.000 \\
Apple & 8.8870 & 0.003 \\
Suning & 97.153 & 0.000 \\
CNPC & 21.733 & 0.000 \\
EEM & 31.297 & 0.000 \\
SPY & 67.920 & 0.000 \\
\hline
\end{tabular}

TABle 7: Parameters for marginal distributions of the six financial assets.

\begin{tabular}{lcccccc}
\hline Parameters & China Bonds 0303 & Apple & Suning & CNPC & EEM & SPY \\
\hline$u$ & 0.000002 & 0.000595 & 0.000334 & -0.000354 & 0.000121 & 0.000353 \\
$\beta_{0}$ & 0.000002 & 0.000001 & 0.000003 & 0.000001 & 0.000009 & 0.000001 \\
$\beta_{1}$ & 0.148630 & 0.116321 & 0.103883 & 0.116107 & 0.168888 & 0.173155 \\
$\beta_{2}$ & 0.850050 & 0.851837 & 0.908557 & 0.890285 & 0.778070 & 0.816123 \\
$\theta$ & 2.877364 & 3.557054 & 4.016967 & 3.844891 & 13.74245 & 4.535059 \\
\hline
\end{tabular}

TABLE 8: The correlation coefficient matrix.

\begin{tabular}{lcccccc}
\hline & China Bonds 0303 & Apple & Suning & CNPC & EEM & SPY \\
\hline China Bonds 0303 & 1 & 0.0431 & 0.0477 & 0.0361 & -0.0159 & 0.0445 \\
Apple & 0.0431 & 1 & -0.0139 & -0.0048 & -0.0243 & 0.5300 \\
Suning & 0.0477 & -0.0139 & 1 & 0.0514 & -0.0827 & -0.0207 \\
CNPC & 0.0361 & -0.0048 & 0.0514 & 1 & -0.0097 \\
EEM & -0.0159 & -0.0243 & -0.0827 & -0.0097 & 0.0152 \\
SPY & 0.0445 & 0.5300 & -0.0207 & 0.0152 & 0.0304 \\
\hline
\end{tabular}

TABLE 9: Future rate of log return simulation.

\begin{tabular}{|c|c|c|c|c|c|c|}
\hline Simulation & China Bonds 0303 & Apple & Suning & CNPC & EEM & SPY \\
\hline 1 & 0.00819 & 0.013112 & -0.00169 & 0.008535 & -0.0086 & 0.007635 \\
\hline 2 & -0.01238 & -0.00387 & 0.046417 & -0.00754 & -0.00493 & -0.00246 \\
\hline 3 & -0.00136 & 0.011882 & 0.051426 & -0.00487 & -0.01529 & -0.00297 \\
\hline 4 & 0.000305 & -0.017 & 0.009973 & -0.00741 & 0.0097 & -0.00158 \\
\hline 5 & $9.44 E-05$ & -0.00558 & 0.018114 & -0.00998 & -0.00924 & 0.003495 \\
\hline 6 & -0.0046 & -0.00226 & -0.05829 & -0.0048 & -0.00343 & 0.000552 \\
\hline 7 & -0.00207 & -0.00395 & -0.03073 & -0.04494 & 0.016671 & 0.001207 \\
\hline$\cdots$ & $\cdots$ & $\cdots$ & $\cdots$ & $\cdots$ & $\ldots$ & $\cdots$ \\
\hline$\ldots$ & $\ldots$ & $\ldots$ & $\ldots$ & $\ldots$ & $\ldots$ & $\ldots$ \\
\hline 1000 & 0.000869 & 0.039122 & 0.047992 & -0.00779 & 0.007335 & 0.003642 \\
\hline
\end{tabular}

$\{0.2199,0.1008,0.0020,0.0208,0.0429,0.6139\}$

$*\{0.000002,0.000595,0.000334,-0.000354,0.000121,0.000353\}^{T}=0.0276 \%$.

To sum up, the proposed personalized mean-CVaR portfolio optimization model can recommend a personalized investment portfolio under expected log return of $0.0276 \%$. 


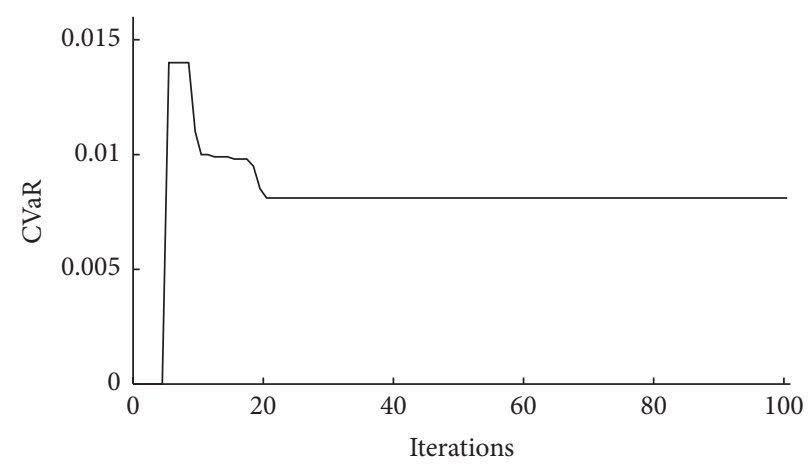

FIgUre 9: Relationship between CVaR and iterations.

Table 10: Optimal investment portfolios for different types of investors.

\begin{tabular}{lcccccccc}
\hline Risk level & China Bonds 0303 & Apple & Suning & CNPC & EEM & SPY & CVaR & Expected return (\%) \\
\hline A & 0.1507 & 0.2900 & 0.0076 & 0.0751 & 0.0841 & 0.3925 & 0.0118 & 0.0384 \\
MA & 0.2199 & 0.1008 & 0.0020 & 0.0208 & 0.0429 & 0.6139 & 0.0089 & 0.0282 \\
M & 0.337059 & 0.111509 & 0.020635 & 0.042033 & 0.131539 & 0.357224 & 0.0084 & 0.0203 \\
MC & 0.358343 & 0.076387 & 0.011917 & 0.031563 & 0.140451 & 0.381338 & 0.0075 & 0.0163 \\
C & 0.465209 & 0.044384 & 0.007992 & 0.024621 & 0.056185 & 0.401609 & 0.0061 & 0.0111 \\
\hline
\end{tabular}

4.2. Experiment 2: Effectiveness of the Personalized Portfolio Optimization Model. In this section, additional experiments are conducted for investors with different risk levels to verify the efficiency of the personalized portfolio optimization model. These experiments are assigned with the same assumptions and dataset with Experiment 1. Table 10 shows optimal investment portfolios for different types of investors. Figure 10 shows optimal weights of financial assets for different types of investors.

The following findings are obtained based on these experimental results.

Firstly, the proposed model can represent investor's risk tolerance. For instance, as shown in Table 10, $\mathrm{CVaR}_{A}>\mathrm{CVaR}_{M A}>\mathrm{CVaR}_{M}>\mathrm{CVaR}_{M C}>\mathrm{CVaR}_{C}$, the value of $\mathrm{CVaR}$ and investor's risk tolerance are negatively correlated.

Secondly, the proposed model can select financial assets with good risk performance. For instance, as shown in Figure 10, the investment ratio of Suning is the smallest, and the investment ratio of SPY Fund is the biggest in optimal portfolios for all kinds of investors.

Finally, the proposed model is able to recommend a personalized portfolio based on investors' risk tolerance. For instance, when investor's risk tolerance decreases, the relative weight of China Bonds 0303 increases, the relative weight of Apple stock decreases, and the weight of other four assets generally maintain steady. Moreover, the investment ratio of Suning is the smallest, and the investment ratio of SPY Fund is the biggest.

To sum up, the proposed mean-CVaR portfolio optimization model is efficient in representing investors' personalized risk tolerance, and hence, it can recommend more personalized investment portfolios for investors.

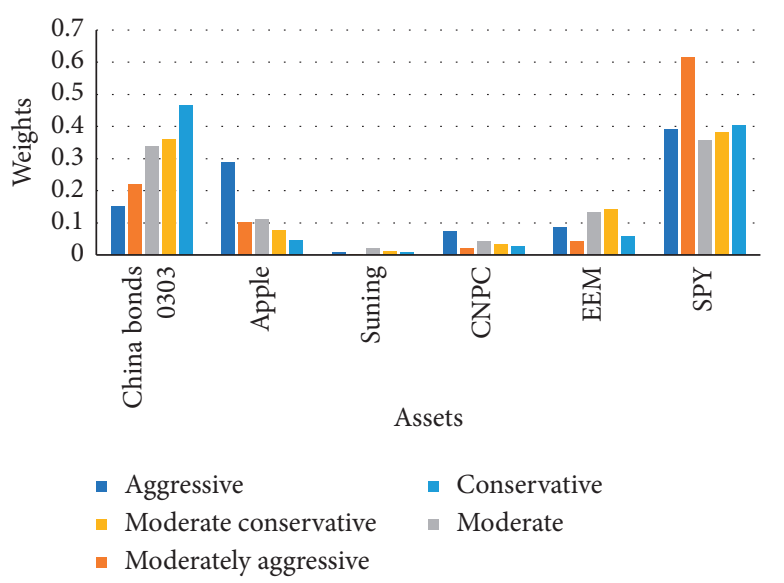

FIgURE 10: Optimal weights of financial assets for different types of investors.

\section{Conclusion}

This research proposes a personalized portfolio optimization model for individual investment which can recommend investment portfolios based on investors' risk tolerance. The model adopts the experts' weights and FCE-integrated method to classify investors' risk tolerance. It adopts the $\mathrm{CVaR}$ to measure the risk of financial assets and uses the combined Copula-GARCH model and Monte Carlo simulation to compute the value of CVaR. This study also applies the mean-CVaR model for individual portfolio optimization and uses the PSO algorithm to determine an optimal portfolio. Experimental results show that the mean-CVaR portfolio optimization model proposed in the research not only can divide the risk tolerance of investors by their demographic characteristics but also can recommend suitable portfolios 
based on their risk tolerance. Moreover, it can effectively help the investors to select high-quality assets' investment and help avoid risk for investors with low risk tolerance.

Future research is recommended to work on the following areas. Firstly, the investors' risk tolerance determination method largely depends on the decision-maker's subjective judgments. A more objective and intelligent risk tolerance determination remains uncompleted. Secondly, the matching rules between investors' risk tolerance and investment assets are determined in advance. Further effort will be conducted to realize a more automatic matching way.

\section{Data Availability}

All data used to support the findings of this study are from the Wind database. The article also includes the data used to support the findings of this study. However, additional data will be made available from the corresponding author upon request.

\section{Conflicts of Interest}

The authors declare that they have no conflicts of interest.

\section{References}

[1] H. M. Holzhauer, X. Lu, R. McLeod, and J. Wang, "RiskTRACK: the five-factor model for measuring risk tolerance," The Journal of Risk Finance, vol. 17, no. 4, pp. 428-445, 2016.

[2] S. Wasiuzzaman and S. Edalat, "Personality, risk tolerance and social network use: an exploratory study," Managerial Finance, vol. 42, no. 6, pp. 536-552, 2016.

[3] H. Markowitz, "Portfolio selection," The Journal of Finance, vol. 7, no. 1, pp. 77-91, 1952.

[4] K. T. Lwin, R. Qu, and B. L. MacCarthy, "Mean-VaR portfolio optimization: a nonparametric approach," European Journal of Operational Research, vol. 260, no. 2, pp. 751-766, 2017.

[5] S. Banihashemi and S. Navidi, "Portfolio performance evaluation in Mean-CVaR framework: a comparison with nonparametric methods value at risk in Mean-VaR analysis," Operations Research Perspectives, vol. 4, pp. 21-28, 2017.

[6] H. Yao, Z. Li, and Y. Lai, "Mean-CVaR portfolio selection: a nonparametric estimation framework," Computers \& Operations Research, vol. 40, no. 4, pp. 1014-1022, 2013.

[7] M. S. Strub, D. Li, X. Cui, and J. Gao, "Discrete-time meanCVaR portfolio selection and time-consistency induced term structure of the CVaR," Journal of Economic Dynamics and Control, vol. 108, Article ID 103751, 2019.

[8] J. E. Grable, "Financial risk tolerance and additional factors that affect risk taking in everyday money matters," Journal of Business and Psychology, vol. 14, no. 4, pp. 625-630, 2000.

[9] R. Alonso-Redondo, E. De Paz, E. Alonso-Herrero, M.-E. García-González, and E. Alfaro-Saiz, "A new method for calculating risk tolerance in the assessment of threatened flora," Journal for Nature Conservation, vol. 21, no. 6, pp. 414-422, 2013.

[10] M. Kannadhasan, "Retail investors' financial risk tolerance and their risk-taking behaviour: the role of demographics as differentiating and classifying factors," Indian Institute of Management Bangalore Management Review, vol. 27, no. 3, pp. 175-184, 2015.

[11] S. A. Raza and S. Rathinam, "A risk tolerance analysis for a joint price differentiation and inventory decisions problem with demand leakage effect," International Journal of Production Economics, vol. 183, pp. 129-145, 2017.

[12] N. P. B. Bollen and S. Posavac, "Gender, risk tolerance, and false consensus in asset allocation recommendations," Journal of Banking \& Finance, vol. 87, pp. 304-317, 2018.

[13] V. A. Subramaniam and T. Athiyaman, "The effect of demographic factors on investor's risk tolerance," International Journal of Commerce and Management Research, vol. 2, no. 3, pp. 136-142, 2016.

[14] E. K. Sulaiman, "An empirical analysis of financial risk tolerance and demographic features of individual investors," Procedia Economics and Finance, vol. 2, pp. 109-115, 2012.

[15] S. Feng and L. D. Xu, "Decision support for fuzzy comprehensive evaluation of urban development," Fuzzy Sets and Systems, vol. 105, no. 1, pp. 1-12, 1999.

[16] J.-F. Chen, H.-N. Hsieh, and Q. H. Do, "Evaluating teaching performance based on fuzzy AHP and comprehensive evaluation approach," Applied Soft Computing, vol. 28, pp. 100-108, 2015.

[17] X. K. Li, X. M. Wang, and L. Lei, "The application of an ANPFuzzy comprehensive evaluation model to assess lean construction management performance," Engineering, Construction and Architectural Management, vol. 27, no. 2, pp. 356-384, 2019.

[18] Y. Mei, J. Ye, and Z. Zeng, "Entropy-weighted ANP fuzzy comprehensive evaluation of interim product production schemes in one-of-a-kind production," Computers \& Industrial Engineering, vol. 100, pp. 144-152, 2016.

[19] Q. Xu, Y. Zhou, C. Jiang, K. Yu, and X. Niu, "A large CVaRbased portfolio selection model with weight constraints," Economic Modelling, vol. 59, pp. 436-447, 2016.

[20] A. E. B. Lim, J. G. Shanthikumar, and G.-Y. Vahn, "Conditional value-at-risk in portfolio optimization: coherent but fragile," Operations Research Letters, vol. 39, no. 3, pp. 163-171, 2011.

[21] M. Karmakar, "Dependence structure and portfolio risk in Indian foreign exchange market: a GARCH-EVT-Copula approach," The Quarterly Review of Economics and Finance, vol. 64, pp. 275-291, 2017.

[22] S. Zhu and M. Fukushima, "Worst-case conditional value-atrisk with application to robust portfolio management," $O p$ erations Research, vol. 57, no. 5, pp. 1155-1168, 2009.

[23] T. Bollerslev, "Generalized autoregressive conditional heteroskedasticity," Journal of Econometrics, vol. 31, no. 3, pp. 307-327, 1986.

[24] S. D. Bekiros and C. G. H. Diks, “The relationship between crude oil spot and futures prices: cointegration, linear and nonlinear causality," Energy Economics, vol. 30, no. 5, pp. 2673-2685, 2008.

[25] V. Ranković, M. Drenovak, B. Urosevic, and R. Jelic, "Meanunivariate GARCH VaR portfolio optimization: actual portfolio approach," Computers \& Operations Research, vol. 72, pp. 83-92, 2016.

[26] I. Kakouris and B. Rustem, "Robust portfolio optimization with copulas," European Journal of Operational Research, vol. 235, no. 1, pp. 28-37, 2014.

[27] X. He and P. Gong, "Measuring the coupled risks: a copulabased CVaR model," Journal of Computational and Applied Mathematics, vol. 223, no. 2, pp. 1066-1080, 2009.

[28] L. J. Hong and G. Liu, "Simulating sensitivities of conditional value at risk," Management Science, vol. 55, no. 2, pp. 281-293, 2009.

[29] J. S. Mehlitz and B. R. Auer, "A Monte Carlo evaluation of non-parametric estimators of expected shortfall," The Journal of Risk Finance, vol. 21, no. 4, pp. 355-397, 2020. 
[30] H.-H. Chen and C.-B. Yang, "Multiperiod portfolio investment using stochastic programming with conditional value at risk," Computers \& Operations Research, vol. 81, pp. 305-321, 2017.

[31] A. İ. Mahmutoğulları, Ö. Çavuş, and M. S. Aktürk, "Bounds on risk-averse mixed-integer multi-stage stochastic programming problems with mean-CVaR," European Journal of Operational Research, vol. 266, no. 2, pp. 595-608, 2018.

[32] A. A. Najafi and S. Mushakhian, "Multi-stage stochastic mean-semivariance-CVaR portfolio optimization under transaction costs," Applied Mathematics and Computation, vol. 256, pp. 445-458, 2015.

[33] T. Zhang and Z. Liu, "Fireworks algorithm for mean-VaR/ CVaR models," Physica A: Statistical Mechanics and Its Applications, vol. 483, pp. 1-8, 2017.

[34] J. Kennedy and R. Eberhart, "Particle swarm optimization," in Proceedings of the IEEE International Conference on Robotics and Automation, Nagoya, Japan, June 1995.

[35] R. Poli, J. Kennedy, and T. Blackwell, "Particle swarm optimization," Swarm Intelligence, vol. 1, no. 1, pp. 33-57, 2007.

[36] L. Feng, X. Zhu, and X. Sun, "Assessing coastal reclamation suitability based on a fuzzy-AHP comprehensive evaluation framework: a case study of Lianyungang, China," Marine Pollution Bulletin, vol. 89, no. 1-2, pp. 102-111, 2014.

[37] R. E. Engle, "Autoregressive conditional heteroskedasticity with estimates of the variance of United Kingdom inflation," Econometrica, vol. 50, no. 50, pp. 987-1008, 1982.

[38] J.-J. Huang, K.-J. Lee, H. Liang, and W.-F. Lin, "Estimating value at risk of portfolio by conditional copula-GARCH method," Insurance: Mathematics and Economics, vol. 45, no. 3, pp. 315-324, 2009.

[39] Z.-R. Wang, X.-H. Chen, Y.-B. Jin, and Y.-J. Zhou, "Estimating risk of foreign exchange portfolio: using VaR and CVaR based on GARCH-EVT-Copula model," Physica A: Statistical Mechanics and Its Applications, vol. 389, no. 21, pp. 4918-4928, 2010. 